\title{
On the Uniform Sampling of CIELAB Color Space and the Number of Discernible Colors
}

\author{
Jean-Baptiste Thomas ${ }^{1}$, Philippe Colantoni ${ }^{2}$, and Alain Trémeau ${ }^{3}$ \\ 1 Université of Bourgogne, Laboratoire d'Electronique, Informatique et Image \\ (LE2I), Dijon, France \\ jean-baptiste.thomas@u-bourgogne.fr \\ ${ }^{2}$ Université Jean Monnet, Centre Interdisciplinaire d'Etudes et de Recherches sur \\ l'Expression Contemporaine, Saint-Étienne, France \\ 3 Université Jean Monnet, Laboratoire Hubert Curien, Saint-Étienne, France
}

\begin{abstract}
This paper presents a useful algorithmic strategy to sample uniformly the CIELAB color space based on close packed hexagonal grid. This sampling scheme has been used successfully in different research works from computational color science to color image processing. The main objective of this paper is to demonstrate the relevance and the accuracy of the hexagonal grid sampling method applied to the $C I E L A B$ color space. The second objective of this paper is to show that the number of color samples computed depends on the application and on the color gamut boundary considered. As demonstration, we use this sampling to support a discussion on the number of discernible colors related to a JND.
\end{abstract}

Keywords: Sampling, 3D close packed hexagonal grid, perceptually uniform color space, computational color imaging.

\section{Introduction}

CIELAB color space 3] has been accepted by the CIE (International Commission on Illumination) as a perceptually pseudo-uniform color space. As such, the Euclidean distance between two specified colors in this space is proportional to the color difference between these two colors perceived by a standard observer. Although this color space has been defined only for very well defined and limited colorimetric conditions, it has been successfully used in practice in many applications in color image processing or computational color science.

Sampling a color space is a major issue in many applications in terms of hardware complexity and speed, accountability to perception, and resulting image quality 8. Historically, a parallelipipedic grid was used for sampling this space 11. Such a grid is defined by a regular lattice that is reproduced over and over in order to fill the space. In some cases, the sampling is performed in an $R G B$ or $C M Y$ space and then transformed into $C I E L A B$, which leads to a large non-uniformity of the final sampling [1930, see Table 1 Table 2, and Figure 1.

S. Tominaga, R. Schettini, and A. Trémeau (Eds.): CCIW 2013, LNCS 7786, pp. 53-67, 2013.

(C) Springer-Verlag Berlin Heidelberg 2013 
Table 1. An indirect sampling of $C I E L A B$ color space from an uniform sampling of a linear $R G B$ induces non-uniformities. The minimum, maximum and average distances between two adjacent samples in the CIELAB color space depend on the sampling step of $R G B$ color space [30]. In this example, the $R G B$ space is coded on 8-bits. Calculations are done with the standard illuminant CIE C. The JND proposed in this article is of $0.2 \Delta E_{a b}^{*}$ units.

\begin{tabular}{|c|c|c|c|c|}
\hline $\begin{array}{c}\text { Sampling } \\
\text { step }\end{array}$ & $\begin{array}{c}\text { Min } \\
\Delta E_{a b}^{*}\end{array}$ & $\begin{array}{c}\text { Max } \\
\Delta E_{a b}^{*}\end{array}$ & $\begin{array}{c}\text { Mean } \\
\Delta E_{a b}^{*}\end{array}$ & $\begin{array}{c}\text { Percent of } \Delta E_{a b}^{*} \\
\text { inf. to 0.2 }\end{array}$ \\
\hline \hline 1 & 0.152 & 13.210 & 0.633 & 1.63 \\
\hline 0.5 & 0.076 & 6.660 & 0.316 & 39.54 \\
\hline 0.25 & 0.038 & 3.340 & 0.157 & 80.83 \\
\hline
\end{tabular}

Table 2. An indirect sampling of $C I E L A B$ color space from an uniform sampling of a non-linear $s R G B$ color space induces non-uniformities too. The minimum, maximum and average distances between two adjacent samples in the CIELAB color space depend on the sampling step of $s R G B$ color space. In this example, we computed these differences within the $s R G B$ space coded on $6,7,8,9$ and 10-bits with different perceptual metrics $\left(\Delta E_{a b}^{*}, \Delta E_{94}^{*}, \Delta E_{00}^{*}\right)$. Calculations are done with the standard illuminant CIE D65. We can notice less non-uniformity than shown in Table 1 because of the gamma correction involved in the $s R G B$ transform.

\begin{tabular}{|c|c|c|c|c|c|c|}
\hline & Bits number & 6 & 7 & 8 & 9 & 10 \\
\cline { 2 - 7 } & dataset size & $64^{3}$ & $127^{3}$ & $256^{3}$ & $512^{3}$ & $1024^{3}$ \\
\hline \hline$\Delta E_{a b}^{*}$ & Mean & 1.9134 & 0.9474 & 0.4714 & 0.2351 & 0.1174 \\
\cline { 2 - 7 } & Min & 0.0075 & 0.0016 & 0.0003 & 0.0 & 0.0 \\
\cline { 2 - 7 } & Max & 5.3717 & 2.7098 & 1.3752 & 0.6973 & 0.3561 \\
\hline \hline \multirow{4}{*}{$E_{94}^{*}$} & Mean & 1.0286 & 0.509 & 0.2532 & 0.1263 & 0.0631 \\
\cline { 2 - 7 } & Min & 0.0025 & 0.0005 & 0.0001 & 0.0 & 0.0 \\
\cline { 2 - 7 } & Max & 4.5906 & 2.2797 & 1.1554 & 0.585 & 0.2997 \\
\hline \hline \multirow{3}{*}{$E_{00}^{*}$} & Mean & 0.9828 & 0.4867 & 0.2421 & 0.1208 & 0.0603 \\
\cline { 2 - 7 } & Min & 0.0024 & 0.0005 & 0.0 & 0.0 & 0.0 \\
\cline { 2 - 7 } & Max & 5.6559 & 2.8403 & 1.4409 & 0.7435 & 0.3862 \\
\hline
\end{tabular}

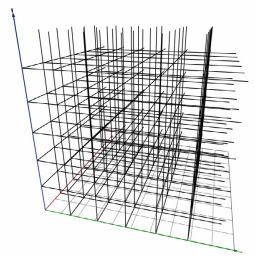

(a) Regular sampling of $s R G B$.

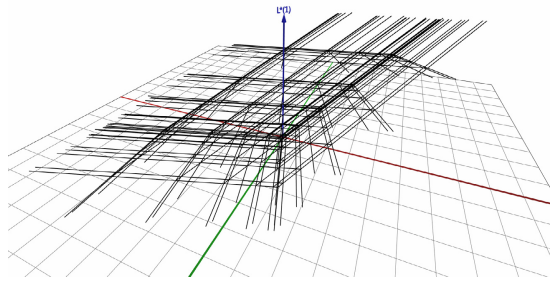

(b) Transform of a regular sampling from $s R G B$ to $C I E L A B$.

Fig. 1. Visualization of a regular sampling of sRGB and its conversion into CIELAB space. We can notice a large lack of uniformity in such a sampling. 
In other cases, the space is directly sampled using a parallelipipedic grid, which has the default property of not being really uniform. For instance, in the case of a cubic sampling using edges of size $a$, the distance between a sample and its closest neighbors can be either $a, a \sqrt{2}$ or $a \sqrt{3}$.

If we could define a Just Noticeable Difference (JND) between color samples as a given Euclidean distance in $C I E L A B$, then we would like to sample it in the most efficient way, i.e. to have the same JND between each sample and its neighbors.

Through this paper we want to show the advantages of using a regular sampling of the CIELAB color space in providing details, issues and algorithm implementation. We want also to provide answers to the following questions: how many discernible colors can be defined in the CIELAB color space uniformly spaced?

In section 2, we first recall the history of this sampling, then in section 3 we explicit the sampling strategy proposed and provide formula and corresponding algorithm in pseudo-code. This sampling has been used successfully in different works related to computational color imaging. Some examples are given and commented in section 4 . Next, in section 5 , we propose a strategy to compute the number of discernible colors. We continue, in section 6 , with a discussion and provide some results in order to demonstrate the relevance and the accuracy of the hexagonal grid sampling method applied to the CIELAB color space. Finally, we conclude this work in pointing out the need of a color space definition with non-Euclidean perceptual metrics.

\section{History and Strategy}

In 1611, Johannes Kepler proposed a mathematical conjecture concerning the densest way to arrange same sized spheres in a $3 D$ Euclidean space. Following that conjecture, a way to obtain the most compact arrangement is to arrange spheres in order to form a face-centered cubic distribution [15]. During the $19^{\text {th }}$ century, Gauss demonstrated that the most compact way to arrange discs in a $2 D$ plan, could be reached if these discs are arranged in a hexagonal way. His demonstration is based on the fact that the center of each disc is at equal distance of its six direct neighbors. He kept open the possibility that a random arrangement can be more compact [7]. Fejes proved that it is the only densest way in the $20^{\text {th }}$ century 29 .

In 1998, Thomas Hales exhaustively demonstrated that spheres arranged in a face-centered cubic or in a hexagonal closed distribution yield the highest density 9].

Based on these works and on the fact that the sphere centers are at equal distance from their direct neighbors, one can define a regular sampling of a tridimensional Euclidean space, such as CIELAB. Such a sampling has been used already in the field of computational color science and color imaging. Firstly it has been used for color specification, such as Munsell re-annotation [32] and OSA color system arrangement 6[17]. Secondly, and specifically, for the sampling 
of $C I E L A B$ color space, it has been used for color image quantization and description [28 27/5] and for display color characterization [24/25/4].

\section{Formula and Algorithm}

\subsection{Sampling Strategy}

A 3D color space, including the gamut of a given device, the spectrum locus under a given illumination, or the gamut of an image, can be sampled in the $C I E L A B$ color space, using a hexagonal closed packing scheme. We distribute samples in the CIELAB color space, inside the considered solid, as if they were the center of spheres in a close-packing of spheres problem. We do not use the face-centered cubic lattices for algorithmic simplicity. This last arrangement would not be of benefit for color imaging applications since we do not use the properties of periodicity and symmetry of such a structure, while the sampling remains the same (we do not discard the fact that for some color specification spaces or other applications, these properties could be useful).

In the algorithmic strategy we propose here, we use only a two layers alternative such as illustrated in Figure 2, creating a hexagonal closed lattice. It is enough to perform a translation to switch from the first layer to the second, and so on. Then, each sphere center is at equal distance of its direct neighbors, which form a Johnson polyhedron number $27\left(J_{27}\right)$, i.e. a triangular orthobicupola [12].

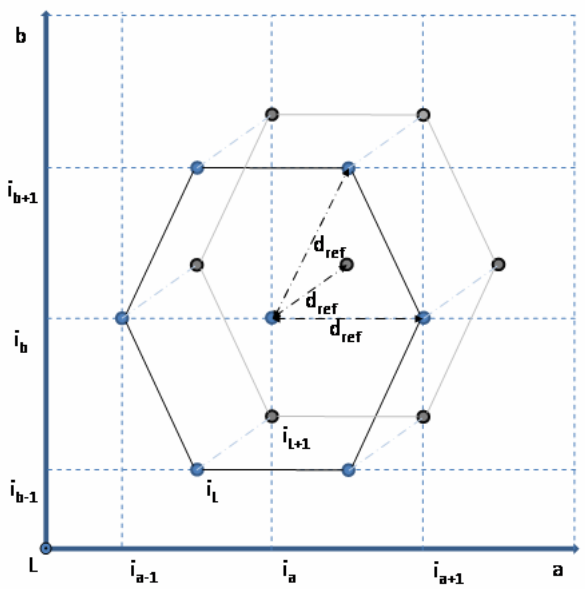

Fig. 2. Sampling scheme of $C I E L A B$ color space. The hexagon drawn in dark plain lines is defined along the $\mathrm{L}^{*}$ axis by a first layer meanwhile the second hexagon drawn in light plain lines corresponds to a second layer at the distance $d_{r e f}$ of the first layer. 


\subsection{Computation}

In this section we explain the sampling algorithm, such as it has been used in 24:28:5].

Let us introduce the following notations: $L_{x}^{*}, a_{x}^{*}, b_{x}^{*}$ denote the coordinates of a given color in the $C I E L A B$ color space, and $L_{y}^{*}, a_{y}^{*}, b_{y}^{*}$ the coordinates of a second one. The CIE $\Delta E_{a b}^{*}$ color distance between these two colors corresponding to the Euclidean distance is defined such as:

$$
\Delta E_{a b}^{*}=\sqrt{d_{L}^{2}+d_{a}^{2}+d_{b}^{2}}
$$

with $\left|L_{x}^{*}-L_{y}^{*}\right|=d_{L},\left|a_{x}^{*}-a_{y}^{*}\right|=d_{a},\left|b_{x}^{*}-b_{y}^{*}\right|=d_{b}$.

Let $d_{\text {ref }}$ be an arbitrary distance in $C I E L A B$ color space between two adjacent samples, which is named as the sampling distance. If we set that the two samples belong to the same layer along the $\mathrm{L}^{*}$ axis, i.e. $d_{L}=0$, and that $d_{a}=d_{\text {ref }}$ and $d_{b}=0$, then $\sqrt{d_{L}^{2}+d_{a}^{2}+d_{b}^{2}}=d_{r e f}$. Likewise, if we set that the two samples belong to the same layer along the $\mathrm{L}^{*}$ axis, i.e. $d_{L}=0$, and that $d_{a}=\frac{1}{2} \times d_{\text {ref }}$ and $d_{b}=\sqrt{\frac{3}{4}} \times d_{\text {ref }}$, then $\sqrt{d_{L}^{2}+d_{a}^{2}+d_{b}^{2}}=d_{r e f}$. Finally, if we set $d_{a}=\frac{1}{2} \times d_{r e f}, d_{b}=\frac{1}{2 \sqrt{3}} \times d_{r e f}$ and $d_{L}=\sqrt{\frac{2}{3}} \times d_{r e f}$, then $\sqrt{d_{L}^{2}+d_{a}^{2}+d_{b}^{2}}=d_{r e f}$.

Let us now set $L_{\min }, L_{\max }, a_{\min }, a_{\max }, b_{\min }$, and $b_{\max }$ the lower and upper gamut color values of the $C I E L A B$ color space along the $L^{*}, a^{*}$ and $b^{*}$ axis.

Considering the arrangement explained above, the $3 D$ grid is then defined such as (See Figure 2):

- if the distance which separates two consecutive samples along the $a^{*}$ axis is such as $d_{r e f}=\left|a_{i_{a}}^{*}-a_{i_{a+1}}^{*}\right|$ then the distance which separates two samples along this axis is $\sqrt{\left(a_{i_{a}}^{*}-a_{i_{a+1}}^{*}\right)^{2}}=d_{\text {ref }}$.

- if the distances which separate two adjacent samples along the $\mathrm{a}^{*}$ and $\mathrm{b}^{*}$ axis are such as $\frac{1}{2} \times d_{\text {ref }}=\left|a_{i_{a}, i_{b}}^{*}-a_{i_{a}, i_{b+1}}^{*}\right|$ and $\sqrt{\frac{3}{4}} \times d_{\text {ref }}=\mid b_{i_{a}, i_{b}}^{*}-$ $b_{i_{a}, i_{b+1}}^{*} \mid$, then the distance which separates two samples in the $\mathrm{a}^{*} \mathrm{~b}^{*}$ plane is $\sqrt{\left(a_{i_{a}, i_{b}}^{*}-a_{i_{a}, i_{b+1}}^{*}\right)^{2}+\left(b_{i_{a}, i_{b}}^{*}-b_{i_{a}, i_{b+1}}^{*}\right)^{2}}=d_{\text {ref }}$.

- the distances which separate two adjacent samples along the $a^{*}, b^{*}$ and $L^{*}$ axis are such as $\frac{1}{2} \times d_{\text {ref }}=\left|a_{i_{L}, i_{a}, i_{b}}^{*}-a_{i_{L+1}, i_{a}, i_{b}}^{*}\right|$ and $\frac{1}{2 \sqrt{3}} \times d_{\text {ref }}=\mid b_{i_{L}, i_{a}, i_{b}}^{*}-$ $b_{i_{L+1}, i_{a}, i_{b}}^{*} \mid$, or $\left|a_{i_{L}, i_{a}, i_{b}}^{*}-a_{i_{L+1}, i_{a}, i_{b}}^{*}\right|=0, \sqrt{\frac{1}{3}} \times d_{\text {ref }}=\left|b_{i_{L}, i_{a}, i_{b}}^{*}-b_{i_{L+1}, i_{a}, i_{b}}^{*}\right|$ and $\sqrt{\frac{2}{3}} \times d_{r e f}=\left|L_{i_{L}, i_{a}, i_{b}}^{*}-L_{i_{L+1}, i_{a}, i_{b}}^{*}\right|$, then the distance which separates these two samples in $C I E L A B$ color space is

$$
d_{\text {ref }}=\left(\left(a_{i_{L}, i_{a}, i_{b}}^{*}-a_{i_{L+1}, i_{a}, i_{b}}^{*}\right)^{2} \cdots+\left(b_{i_{L}, i_{a}, i_{b}}^{*}-b_{i_{L+1}, i_{a}, i_{b}}^{*}\right)^{2}+\left(L_{i_{L}, i_{a}, i_{b}}^{*}-L_{i_{L+1}, i_{a}, i_{b}}^{*}\right)^{2}\right)^{1 / 2}
$$


The smaller $d_{r e f}$ is the finer the sampling of the color space is, then the generated number of samples increases inversely proportionally to the distance $d_{\text {ref }}$.

Thus, if we consider: $N_{L}=\frac{L_{\max }-L_{\min }}{d_{\text {ref }} \sqrt{\frac{2}{3}}}, N_{a}=\frac{a_{\max }-a_{\min }}{d_{\text {ref }}}$ and $N_{b}=\frac{b_{\max }-b_{\min }}{d_{\text {ref }} \sqrt{\frac{3}{4}}}$, with $N_{L}, N_{a}$ and $N_{b}$ the number of sample values generated along the $L^{*}, a^{*}$ and $b^{*}$ axis respectively. Then, $N=N_{L} \times N_{a} \times N_{b}$, the number of samples is function of $\frac{1}{\frac{1}{\sqrt{2}} \times d_{r e f}^{3}}$.

The final number of patches is constituted of the intersection of the sampled cube with the gamut we want to sample.

\subsection{Algorithm}

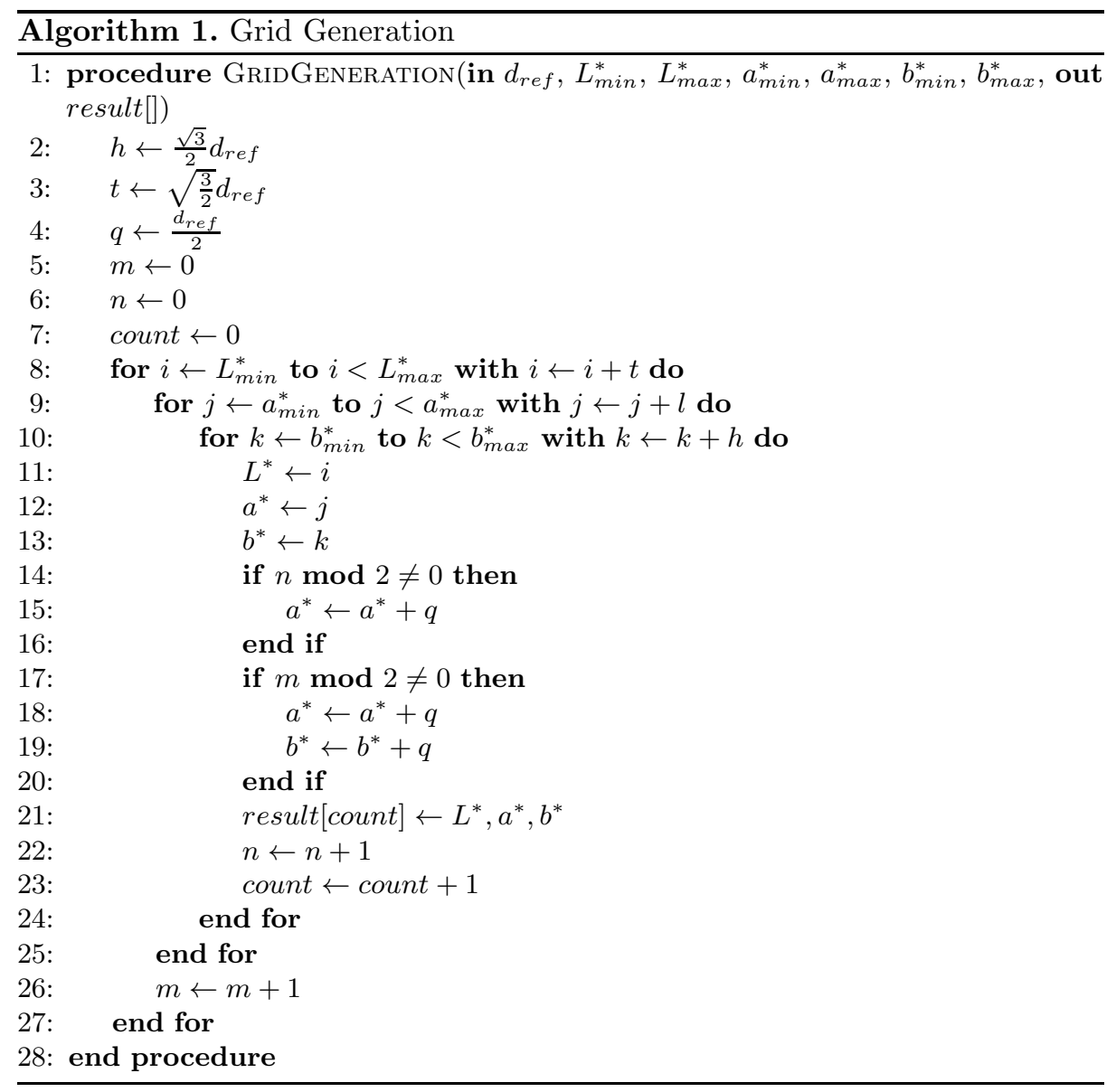


In this section, we explicit the algorithm. The sampling function depends of the upper and lower boundaries of each axis and of $d_{r e f}$. It creates as output an array that contains the list of obtained samples (See Algorithm 1).

\section{An Application Dependent Problem}

In the following we give some examples of applications for which such a sampling is useful. These examples are related to: LUT interpolation, quantization and visualization. These examples show that the number of discernible colors depends of the application.

- Color image quantization.

A uniform quantization algorithm has been proposed using this sampling, giving good results compared with other methods 28. This scheme was based on a split and merge process based on simple weighting indicators (Figure 3).

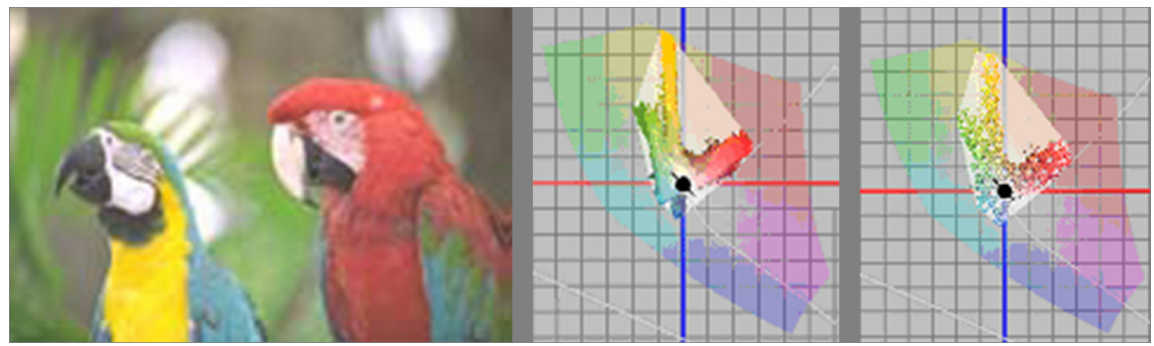

Fig. 3. Visualization of the color gamut of an image. On the left, the image, in the middle the gamut of the image, on the right the image gamut sampled with the presented scheme.

In 28] it is demonstrated that the quality of the quantization depends firstly of the number of colors desired. It is also demonstrated that with a $d_{r e f}=$ 2 or a $d_{r e f}=3$ in $C I E L A B$ we can obtain a good compromise between sampling precision and image quality, and that the choice of $d_{r e f}$ value, is before all, image dependent.

- Color image characterization and visualization for cultural heritage.

For the study of art painting, this sampling is of great help to visualize the color content of calibrated images of masterpieces [5].

Indeed, visualization of the color content of a painting can help to better understand the style, compositional structure and material content. There are several ways to visualize colorimetric data from a color image. One option is to use 3D Virtual Reality to view colorimetric data in an arbitrary orientation in a standard color space. Based on this sampling, a new colorimetric visualization method has been proposed [5]. The originality of this 

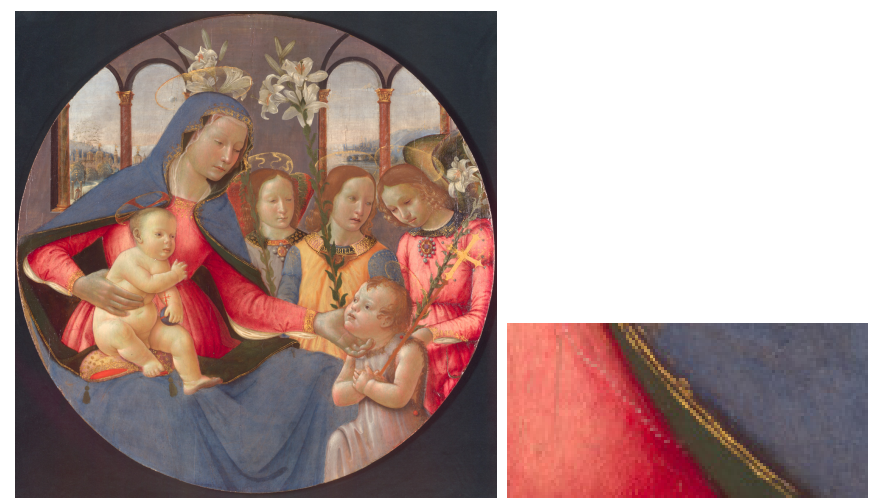

(a) Image considered on the left and a sample on it on the right.

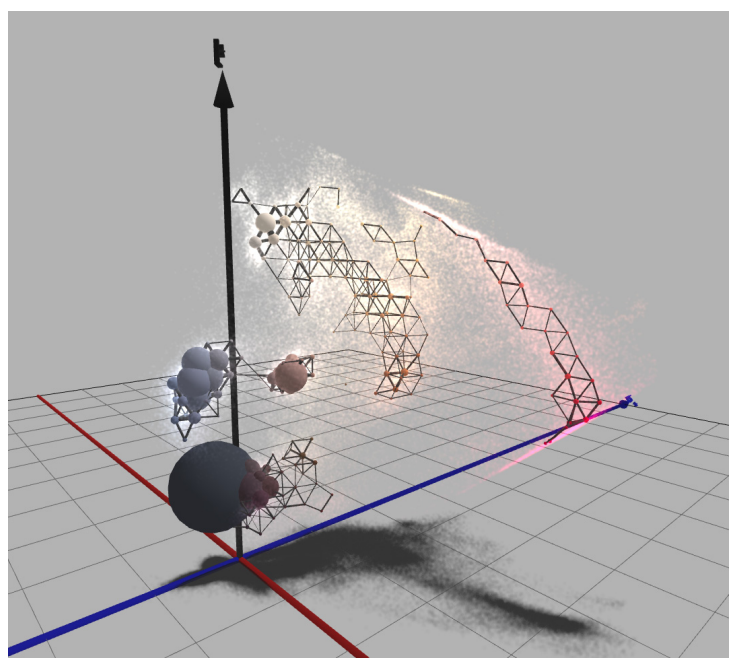

(b) Visualization of the color information from the image sample above.

Fig. 4. Visualization of the color content of a calibrated picture of Virgin and Child with Saint John the Baptist and Three Angels by Sebastiano Mainardi. We can see the color gamut of the image (the color data clouds), the 3D histogram (the spheres, which radius is proportional to the number of pixels of a given color in the image) and information on the color gradient that links adjacent colors in the image (the width of the tetrahedral structure, more the edges are thick more these colors are spatially closed within the image, showing a color gradient). 
method is that it includes also the spatial organization of the colors within the painting. Thus, it is possible to visualize information on color gradients that may appear in the painting using simple 3D primitives. The efficiency of this method has been shown on a colorimetrically calibrated image representing an Italian Renaissance painting (Figure 4). In this application, the relevance of the method and the number of samples depends firstly of the value $d_{\text {ref }}$ used and secondly of the shape of the gamut considered.

- Display colorimetric characterization.

Device color characterization has been successfully performed while using this sampling [24254]. Results overcome most of today's methods. The best results are obtained when this sampling is used in synergy with an interpolation/approximation method based on polyharmonic splines (Figure 55).

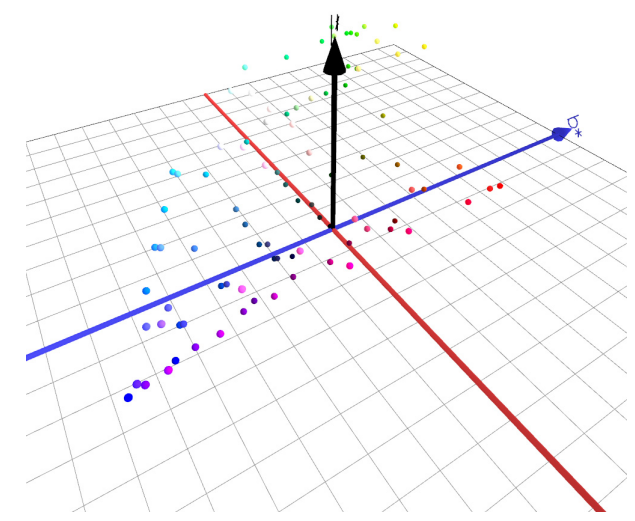

Fig. 5. Visualization of the color gamut of a display sampled with the presented scheme. The gamut boundaries are not shown here but can be easily computed from the data set using any method.

From experiments done in our laboratories with different digital devices, e.g. CRT monitors or LCD monitors, we estimated that with a $d_{\text {ref }}=10$ or a $d_{\text {ref }}=15$ in $C I E L A B$ we can obtain a good compromise between the number of samples to measure in order to characterize a display and the characterization accuracy. We also observed, but not proved, that the number of samples depends firstly of the value $d_{\text {ref }}$ used and secondly of the size of the gamut considered.

We have seen in the three applications mentioned that the number of relevant colors depends firstly of the image studied and/or of the shape and the size of the gamut considered. We can also claim that the number of relevant colors depends strongly on the number of discernible colors, and consequently on the value $d_{r e f}$ used. Let us now consider another kind of application, for which the accuracy of results depends even more on the accuracy of the sampling. 


\section{$5 \quad$ Number of Discernible Colors}

In colorimetry, the discretization of the color gamut of the spectrum locus can be optimized accordingly to a JND. If we consider $d_{r e f}$ as a JND, then we do not need to consider a continuous space anymore, but only the number of samples given by such sampling scheme, as illustrated in Figure 6 .

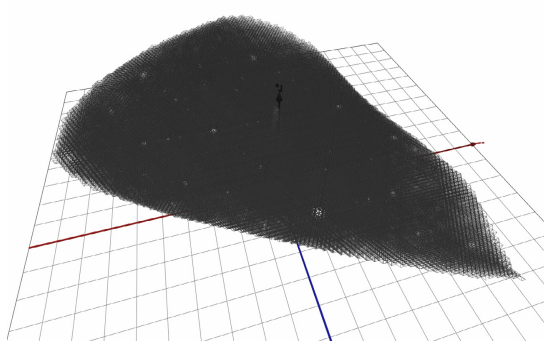

(a) $d_{\text {ref }}$ of $3: 111,933$ selected colors.

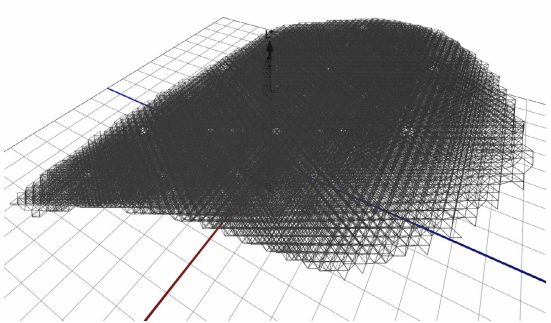

(b) $d_{\text {ref }}$ of 5: 24,178 selected colors

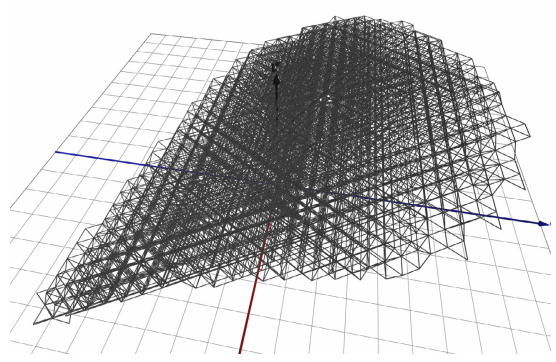

(c) $d_{\text {ref }}$ of 10: 3,025 selected colors

Fig. 6. Visualization of the discretization of the color gamut associated to the spectrum locus of the 1931 standard observer under a D65 standard illumination. Here, we used three different $d_{r e f}$ and obtained three datasets of colors that describe plainly this color gamut, assuming perceptual uniformity with the Euclidean metric and $d_{r e f}$ as the JND.

The parameters of the algorithm method used may be debated as some factors influence the sampling quality. First, parameter $d_{\text {ref }}$ is critical for the sampling, but some other factors play also a role in the final quality. We can notice two major factors: the Color Gamut Boundary (CGB) used and the origin of the sampling process in interaction with the boundaries. The CGB has no direct interaction with the sampling strategy itself. But in a given application, results can be critically different while using one or another CGB in the number of selected data and in the number of occurrences of these data in a given image. So, under estimating the gamut or over estimating the gamut can be seen as a problem. 
The origin of the sampling is also of major importance. For instance, while working with color imaging devices, there is often an offset, which leads the gamut not to be defined in CIELAB coordinates $(0,0,0)$. The darkest point could be defined for instance by $(4,1,2)$, in this case it could be judicious to choose the starting point of the sampling such as $(4,1,2)$ to be sure to have the most useful data related to the darkest part. The same effect can be observed in the brightest part of the gamut defined by the highest coordinates of the gamut shape which should be $\left(100, a_{w}, b_{w}\right)$. But, the last sample included in the data set can be $\left(98, a_{l}, b_{l}\right)$ depending on $d_{\text {ref }}$. The same effect can be observed everywhere around the boundaries. It is then necessary to add an offset parameter from where the sampling shall start. This offset will not be debated more here, but for some application it is of major importance. It can be defined by a priori knowledge on the gamut or/and on the CGB, or refined with several iterations.

The most critical parameter is the choice of $d_{\text {ref }}$. Tremeau et al. [30] demonstrated that a $d_{\text {ref }}=0.2$ in $C I E L A B$ could be considered as a good approximation of the JND. With such a distance the number of samples generated is very high. Intuitively, one would like to find a good tradeoff between the accuracy of the sampling, the sensitivity of the Human Visual System, and hardware complexity and speed.

A good strategy could be to keep $d_{\text {ref }}$ stucks with a JND or to an acceptability threshold based on human vision. In the colorimetric case, several choices can be done. Kang [14 said on the page 167 of his book that the JND is of $1 \Delta E_{a b}^{*}$ unit. Mahy et al. [18] study assessed that the JND is of $2.3 \Delta E_{a b}^{*}$ units. In the case of color imaging devices, many different sets of thresholds have been used [1|10|22|23, considering $\Delta E_{a b}^{*}$, for color imaging devices. Stokes et al. [26] found a perceptibility acceptance for pictorial images of an average of 2.15 units. Catrysse et al. [2] used a threshold of 3 units.

So, what is the best value for $d_{\text {ref }}$ ? To answer to this question, let us consider for example that we want to sample the color gamut associated to the spectrum locus of the 1931 standard observer under a D65 standard illumination (See Figure 6).

\section{Discussion and Results}

If we use a $d_{\text {ref }}$ of 3 , we obtain 111,933 discernible colors. In the same way, we obtain 24,178 colors for a $d_{\text {ref }}$ of 5 , and 3,025 colors for a $d_{\text {ref }}$ of 10 .

With this sampling, both Min, Max and Mean values of $\Delta E$ are equal to $d_{r e f}$ and the variance of the twelve direct neighbors is equal to 0 . These values, compared with the values given in Table1 and Table 2, demonstrate that this sampling is more uniform than the indirect sampling of $C I E L A B$ color space based on an uniform sampling of the $R G B$ color space 1

If we increase the $d_{r e f}$ value, then consequently the number of sample computed decrease. These two parameters are related by a non-linear law shown in Figure 7

${ }^{1}$ The values computed for the indirect sampling of the $C I E L A B$ color space are only computed with 6 neighbors. 


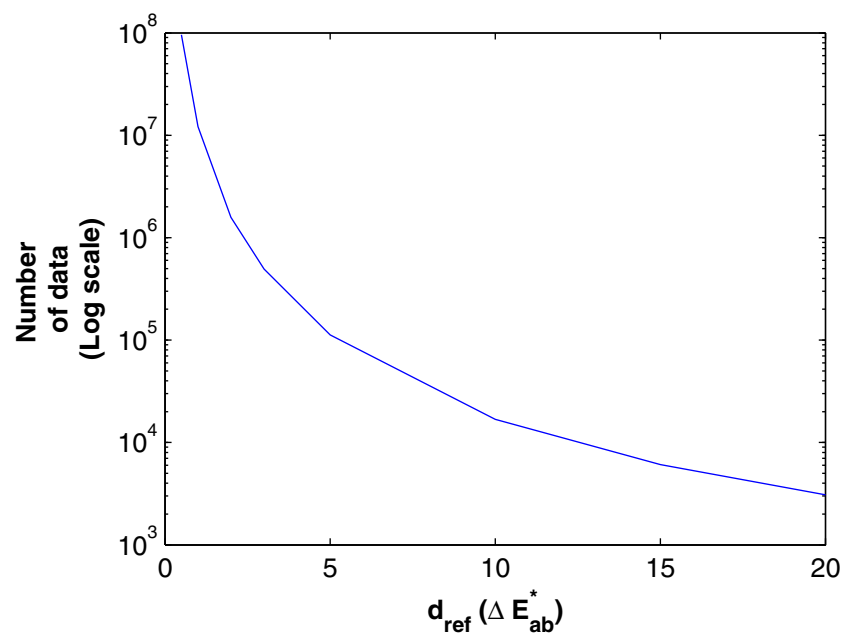

Fig. 7. Relationship between $d_{r e f}$ and the number of samples data for an CIE standard observer 1931 and a D65 illumination

We can compare these results with the number of discernible colors proposed by other authors:

- Judd and Wyszecki [13] who talked of about 10,000,000 colors included into the theoretical limits.

- Pointer and Attridge 21], considering some restriction in the possible natural spectra (the MacAdam limits), who talked of about 2,279,381 colors.

- Linhares at al. [16], based on natural scene analysis, who talked of about $2,275,698$ colors.

Although the first ones used a parallelipipedic grid and the last ones used an analysis based on the CIEDE2000 color difference, the number of discernible colors both proposed is quite consistent.

If we look at our results using $\Delta E_{a b}^{*}$, we can find a number of discernible colors of $12,163,500$ using a JND of 1 units, which is relatively closed to the number given by Judd and Wyszecki. In another hand, the JND of 1 seems to be not very well fitted by $\Delta E_{a b}^{*}$. If we consider a given number of about $2,300,000$ discernible colors, the JND would be of around $1.78 \Delta E_{a b}^{*}$ units while using our sampling method, which is closer to the one proposed by Mahy et al. In all cases, a variation can be tolerated due to the approximation done on the gamut boundaries and on the perceptual uniformity of the couple $\left(C I E L A B, \Delta E_{a b}^{*}\right)$.

Some could argue that does not make sense to define a JND value in the $C I E L A B$ color space because in this color space MacAdam ellipses are, for some of them, up to 3 times larger than the others. Others claim that even if the $C I E L A B$ color space is only approximately uniform, it is nevertheless essential to define a unique JND 
value whatever the colors considered, otherwise it does not make sense to compute a $\Delta E_{a b}^{*}$ to compare any color difference.

Based on the different studies mentioned in this paper and on the computations done, we consider that the best tradeoff, between the number of discernible colors and the JND between them, seems to be a JND between 1.5 and 2. $\Delta E_{a b}^{*}$ units. In taking it the other way around, we could assume that there are, at most, 2,300,000 discernible colors in this color gamut.

Of course this number is not a definite perceptual number of discernible colors, but only an indication of a number of samples used to define a given gamut. Morovic et al. 20] demonstrated that the quest of such a number of visible colors is vain. However, this case studied helps to demonstrate the efficiency of our sampling.

Even if the complexity of the hexagonal grid sampling is slightly higher than with the parallelepiped grid sampling and that the number of samples is lower with the parallelepiped sampling, there is a benefit to use the hexagonal sampling. Indeed, the accuracy of the sampling is greatly increased with the hexagonal grid sampling than with the parallelepiped one.

\section{Conclusion}

We have shown that the uniform sampling strategy detailed in this paper could be of great interest in many applications. However until now it was not widely used in the image processing community, as there was some uncertainties about the number of discernible colors and about the parameter $d_{\text {ref }}$. We have demonstrated in this paper that the number of color samples is dependent of the shape and of the size of the color gamut considered. We have also shown that, for color images, this number is also dependent of the color gamut of the image studied. We have proposed a strategy to optimize this sampling in function of the color gamut boundary. We have demonstrated that the number of discernible color is dependent of the parameter $d_{\text {ref }}$ and that this latter can be set to the JND. Lastly, we have demonstrated the relevance and the accuracy of the hexagonal grid sampling method applied to the $C I E L A B$ color space.

As an extension, we propose to build a similar uniform sampling in using a tabulated version of $C I E L A B$ adapted to perceptual metrics different from the Euclidean one, such as the $\Delta E_{94}, \Delta E_{C M C}, \Delta E_{00}$ and to extend it to other color spaces than the $C I E L A B$ color space, such as color appearance spaces (e.g. JCH color space). Let us note that, to achieve such kind of sampling, it is far more difficult. One can used the tabulated version of CIELAB proposed by Urban et. al [31. We initiated a work based on a different approach in a previous paper [5], and a complete study of the distribution we reached will be presented in a near future communication.

\section{References}

1. Abrardo, A., Cappellini, V., Cappellini, M.: MECOCCI, A.: Art-works colour calibration using the vasari scanner. In: Color Imaging Conference, pp. 94-97. IS \& T The Society for Imaging Science and Technology (1996) 
2. Catrysse, P.B., Wandell, B.A., EI Gamal, A.: Comparative analysis of color architectures for image sensors. In: Sampat, N., Yeh, T. (eds.) Proc. SPIE, vol. 3650, pp. 26-35 (1999)

3. CIE: 015:2004, Colorimetry, 3rd edn. Commission Internationale de l'Eclairage (2004)

4. Colantoni, P., Thomas, J.-B.: A Color Management Process for Real Time Color Reconstruction of Multispectral Images. In: Salberg, A.-B., Hardeberg, J.Y., Jenssen, R. (eds.) SCIA 2009. LNCS, vol. 5575, pp. 128-137. Springer, Heidelberg (2009)

5. Colantoni, P., Thomas, J.B., Pillay, R.: Graph-based 3d visualization of color content in paintings. In: Proceedings of the 11th VAST International Symposium on Virtual Reality, Archaeology and Cultural Heritage, Paris, September 22-24, vol. 2, pp. 25-30 (September 2010)

6. Foss, C.E.: Space lattice used to sample the color space of the committee on uniform color scales of the optical society of america. J. Opt. Soc. Am. 68(11), 1616-1619 (1978)

7. Gauss, C.F.: Disquisitiones arithmeticae. Apud G. Fleischer, Leipzig (1801); reprinted Springer, New-York (1986)

8. Gentile, R.S., Allebach, J.P., Walowit, E.: Quantization of color images based on uniform color spaces. Journal of Imaging Technology 16(1), 11-21 (1990)

9. Hales, T.C.: An overview of the kepler conjecture (1998)

10. Hardeberg, J.: Acquisition and reproduction of colour images: colorimetric and multispectral approaches. These de doctorat, Ecole Nationale Superieure des Telecommunications, ENST, Paris, France (January 1999)

11. Hill, B., Roger, T., Vorhagen, F.W.: Comparative analysis of the quantization of color spaces on the basis of the cielab color-difference formula. ACM Trans. Graph. 16, 109-154 (1997)

12. Johnson, N.W.: Convex solids with regular faces. Canadian Journal of Mathematics 18, 169-200 (1966)

13. Judd, D.B., Wyszecki, G.: Color in business, science, and industry, 3rd edn. Wiley, New York (1975)

14. Kang, H.R. (ed.): Color technology for electronic imaging devices. SPIE Press (1997)

15. Kepler, J.: The six-cornered snowflake. Monograph (1611)

16. Linhares, J.M.M., Pinto, P.D., Nascimento, S.M.C.: The number of discernible colors in natural scenes. J. Opt. Soc. Am. A 25(12), 2918-2924 (2008)

17. MacAdam, D.L.: Colorimetric data for samples of osa uniform color scales. J. Opt. Soc. Am. 68(1), 121-130 (1978)

18. Mahy, M., Eycken, L.V.V., Oosterlinck, A.: Evaluation of uniform color spaces developed after the adoption of cielab and cieluv. Color Research \& Application 19(2), 105-121 (1994)

19. Mahy, M., Van Mellaert, B., Van Eycken, L., Oosterlinks, A.: The influence of uniform color spaces on color image processing: a comparative study of cielab, cieluv, and atd. Journal of Imaging Technology 17(5), 232-243 (1991)

20. Morovic, J., Cheung, V., Morovic, P.: Why we dont know how many colors there are. In: CGIV (May 2012)

21. Pointer, M.R., Attridge, G.G.: The number of discernible colours. Color Research \& Application 23, 52-54 (1998)

22. Schläpfer, K.: Farbmetrik in der reproduktionstechnik und im mehrfarbendruck. In: Schweiz, S.G. (ed.) 2. Auflage UGRA (1993)

23. Stamm, S.: An investigation of color tolerance. In: TAGA Proceedings, pp. 156-173 (1981) 
24. Stauder, J.F., Colatoni, P.F., Blonde, L.F.: Device and method for characterizing a colour device. EP1701555 (September 2006)

25. Stauder, J., Thollot, J., Colantoni, P., Tremeau, A.: Device, system and method for characterizing a colour device. European Patent WO/2007/116077, EP1845703 (October 2007)

26. Stokes, M., Fairchild, M.D., Berns, R.S.: Precision requirements for digital color reproduction. ACM Trans. Graph. 11(4), 406-422 (1992)

27. Thomas, J.B., Chareyron, G., Trémeau, A.: Image watermarking based on a color quantization process. Multimedia Content Access: Algorithms and Systems 6506(1), 650603 (2007)

28. Thomas, J.B., Trémeau, A.: A gamut preserving color image quantization. In: Proceedings of the 14th International Conference of Image Analysis and Processing Workshops, ICIAPW 2007, pp. 221-226. IEEE Computer Society, Washington, DC (2007)

29. Tóth, F.: On the stability of a circle packing. Ann. Univ. Sci. Budapestinensis, Sect. Math. 3-4, 63-66 (1960/1961)

30. Trémeau, A., Konik, H., Lozano, V.: Limits of using a digital color camera for color image processing. In: Proceedings of the IS\&T/OSA Optics \& Imaging in the Information Age, Rochester, New York, October 20-24, pp. 150-155 (October 1996)

31. Urban, P., Schleicher, D., Rosen, M.R., Berns, R.S.: Embedding non-euclidean color spaces into euclidean color spaces with minimal isometric disagreement. Journal of the Optical Society of America A 24(6), 1516-1528 (2007)

32. Wyszecki, G.: A regular rhombohedral lattice sampling of munsell renotation space. Journal of the Optical Society of America 44(9), 725-734 (1954) 